\title{
Perturbation theories for symmetry-protected bound states in the continuum on two-dimensional periodic structures
}

\author{
Lijun Yuan* \\ College of Mathematics and Statistics, Chongqing Technology and Business University, Chongqing, China \\ Ya Yan $\mathrm{Lu}$ \\ Department of Mathematics, City University of Hong Kong, Hong Kong
}

\begin{abstract}
On dielectric periodic structures with a reflection symmetry in a periodic direction, there can be antisymmetric standing waves (ASWs) that are symmetry-protected bound states in the continuum (BICs). The BICs have found many applications, mainly because they give rise to resonant modes of extremely large quality-factors ( $Q$-factors). The ASWs are robust to symmetric perturbations of the structure, but they become resonant modes if the perturbation is non-symmetric. The $Q$-factor of a resonant mode on a perturbed structure is typically $O\left(1 / \delta^{2}\right)$ where $\delta$ is the amplitude of the perturbation, but special perturbations can produce resonant modes with larger $Q$-factors. For twodimensional (2D) periodic structures with a 1D periodicity, we derive conditions on the perturbation profile such that the $Q$-factors are $O\left(1 / \delta^{4}\right)$ or $O\left(1 / \delta^{6}\right)$. For the unperturbed structure, an ASW is surrounded by resonant modes with a nonzero Bloch wave vector. For $2 \mathrm{D}$ periodic structures, the $Q$-factors of nearby resonant modes are typically $O\left(1 / \beta^{2}\right)$, where $\beta$ is the Bloch wavenumber. We show that the $Q$-factors can be $O\left(1 / \beta^{6}\right)$ if the ASW satisfies a simple condition.
\end{abstract}

\section{INTRODUCTION}

In recent years, bound states in the continuum (BICs) have attracted much attention in the photonics community [1 3 . In classical wave systems, a BIC is a trapped or guided mode with a frequency in the frequency interval where outgoing radiative waves exist. Mathematically, a BIC corresponds to a discrete eigenvalue in the continuous spectrum, and causes the non-uniqueness of a corresponding scattering or diffraction problem [4, 5]. Optical BICs have been studied in a number configurations including waveguides with local distortions [6, 7], waveguides with lateral leaky structures $88-10$, waveguides with anisotropic materials [11, and periodic structures surrounded by or sandwiched between homogeneous media [4, 5, 12, 32. On symmetric structures, there may be symmetry-protected BICs that do not couple with the radiative waves due to a symmetry mismatch 4, 5, 12[16. Other BICs exist without any symmetry mismatch, but in some cases, their existence still depends crucially on the symmetry and they are robust against structural perturbations that preserve the symmetry [33 35]. Optical BICs have applications in lasing [36, sensing [37, 38, filtering [39, 40, and switching [41, and for enhancing emissive processes and nonlinear optical effects [42, 43.

A BIC can be considered as a resonant mode with an infinite $Q$-factor. This implies that resonant modes with extremely large $Q$-factors can be created by perturbing the structure or varying a physical parameter slightly [43 47]. Since many applications of the BICs are related to the high- $Q$ resonances, it is of significant importance to understand how the $Q$-factors depend on the structural or parametric perturbations. It is well-known

* Corresponding author: ljyuan@ctbu.edu.cn that symmetry-protected BICs are robust with respect to symmetric perturbations 35. For a non-symmetric perturbation of amplitude $\delta$, the symmetry-protected BICs become resonant modes and their $Q$-factors are typically $O\left(1 / \delta^{2}\right)$ [46. On periodic structures, a BIC is always surrounded by a family of resonant modes depending on the wave vector. For $2 \mathrm{D}$ periodic structures with a $1 \mathrm{D}$ periodicity, if there is a BIC with a Bloch wavenumber $\beta_{*}$, then the resonant mode with Bloch wavenumber $\beta$ (close to $\beta_{*}$ ) typically has a $Q$-factor proportional to $1 /\left|\beta-\beta_{*}\right|^{2}$. In previous works [43, 45, we identified some special BICs such that the $Q$-factors of the nearby resonant modes are at least $O\left(1 /\left|\beta-\beta_{*}\right|^{4}\right)$. It is also known that for some cases, the $Q$-factors are $O\left(1 /\left|\beta-\beta_{*}\right|^{6}\right)[48$.

In this paper, we present new perturbation results for antisymmetric standing waves (ASWs) on 2D periodic structures with a reflection symmetry in the periodic direction. The ASWs are symmetry-protected BICs since they have a symmetry mismatch with plane waves propagating in the normal direction (perpendicular to the periodic direction). For a non-symmetric structural perturbation with amplitude $\delta$ and profile $F$, we show that the $Q$-factor is in general $O\left(1 / \delta^{2}\right)$, but it becomes $O\left(1 / \delta^{4}\right)$ if $F$ satisfies a simple condition involving the wave field of the ASW, and it further becomes $O\left(1 / \delta^{6}\right)$ if in addition to the previous condition, $F$ is anti-symmetric. We also consider the perturbation with respect to the Bloch wavenumber. It is known that for any BIC (with Bloch wavenumber $\beta_{*}$ ) satisfying a special condition [45], the $Q$ factors of nearby resonant modes (with Bloch wavenumber $\beta)$ are $O\left(1 /\left|\beta-\beta_{*}\right|^{4}\right)$ in general. We show that if the $\mathrm{BIC}$ is an ASW, then the $Q$-factors are in fact $O\left(1 / \beta^{6}\right)$. These theoretical results are validated by numerical examples for periodic structures with three arrays of circular dielectric cylinders.

The rest of this paper is organized as follows. In Sec. II. 
a brief introduction is given for BICs and resonant modes on 2D periodic structures. In Sec. III, a detailed structural perturbation theory is developed for ASWs on periodic structures with a reflection symmetry. In Sec. IV. a special wavenumber perturbation theory is briefly presented for ASWs. To validate the perturbation theories, numerical examples are presented in Sec. V. The paper is concluded with a brief discussion in Sec. VI.

\section{BICS AND RESONANT MODES}

In this section, we present the mathematical formulation and give precise definitions for BICs and resonant modes. We consider 2D dielectric structures that are invariant in $z$, periodic in $y$ with period $L$, and bounded in the $x$ direction by $|x|<D$ for some constant $D$, where $\{x, y, z\}$ is a Cartesian coordinate system. Assuming the surrounding medium for $|x|>D$ is vacuum, we have a dielectric function $\epsilon$ satisfying $\epsilon(x, y+L)=\epsilon(x, y)$ for all $(x, y)$, and $\epsilon(x, y)=1$ for $|x|>D$. For the $E$ polarization, the $z$-component of the electric field, denoted as $u$, satisfies the following Helmholtz equation

$$
\frac{\partial^{2} u}{\partial x^{2}}+\frac{\partial^{2} u}{\partial y^{2}}+k^{2} \epsilon u=0
$$

where $k=\omega / c$ is the free space wavenumber, $\omega$ is the angular frequency, $c$ is the speed of light in vacuum, and the time dependence is assumed to be $e^{-i \omega t}$.

A Bloch mode on such a periodic structure is a solution of Eq. (1) given as

$$
u(x, y)=\phi(x, y) e^{i \beta y}
$$

where $\phi$ is periodic in $y$ with period $L$ and $\beta$ is the real Bloch wavenumber. Due to the periodicity of $\phi, \beta$ can be restricted to the interval $[-\pi / L, \pi / L]$. If $\omega$ is real and $\phi \rightarrow 0$ as $|x| \rightarrow \infty$, then $u$ given by Eq. (2) is a guided mode. Typically, guided modes that depend on $\beta$ and $\omega$ continuously can only be found below the light line, i.e., for $k<|\beta|$. A BIC is a special guided mode above the light line, i.e., $\beta$ and $k$ satisfy the condition $k>|\beta|$. For a given structure, BICs can only exist at isolated points in the $\beta-\omega$ plane.

In the homogeneous medium given by $|x|>D$, we can expand a Bloch mode in plane waves, that is

$$
u(x, y)=\sum_{j=-\infty}^{\infty} c_{j}^{ \pm} e^{i\left(\beta_{j} y \pm \alpha_{j} x\right)}, \quad|x|>D
$$

where the "+" and "-" signs are chosen for $x>D$ and $x<-D$ respectively, and

$$
\beta_{j}=\beta+\frac{2 \pi j}{L}, \quad \alpha_{j}=\sqrt{k^{2}-\beta_{j}^{2}} .
$$

If $\omega$ is positive and $k<\left|\beta_{j}\right|$, then $\alpha_{j}=i \sqrt{\beta_{j}^{2}-k^{2}}$ is pure imaginary, and the corresponding plane wave is evanes- cent. For a BIC, one or more $\alpha_{j}$ are real, and the corresponding coefficients $c_{j}^{ \pm}$vanish, since the field must decay to zero as $|x| \rightarrow \infty$.

On periodic structures with a reflection symmetry in the $y$ direction, there may be ASWs which are symmetryprotected BICs with $\beta=0$. Assuming the dielectric function $\epsilon(x, y)$ is even in $y$, then the field $u$ of an ASW is an odd function of $y$. If the ASW is non-degenerate, i.e., the multiplicity is 1 , the real and imaginary parts of $u$ (if they are nonzero) are themselves ASWs of the same frequency, thus they are linearly dependent. Without loss of generality, we assume the field $u$ of a non-degenerate ASW is real. We consider only ASWs with a frequency $\omega$ such that $0<k<2 \pi / L$. In that case, $\alpha_{0}=k$ is real and all other $\alpha_{j}$ for $j \neq 0$ are pure imaginary. Since $u$ is odd in $y$, the coefficients $c_{0}^{ \pm}$are always zero.

On periodic structures, a resonant mode is a Bloch mode with a complex frequency $\omega$ and a real Bloch wavenumber $\beta$ and it radiates out power as $|x| \rightarrow \infty$. The resonant modes form bands, and on each band, they depend on $\beta$ continuously. Under the assumed time dependence, the imaginary part of the complex frequency of a resonant mode must be negative, so that its amplitude can decay with time. The $Q$-factor of a resonant mode can be defined as $Q=-0.5 \operatorname{Re}(\omega) / \operatorname{Im}(\omega)$. The expansion (3) is still valid, but the complex square root for $\alpha_{j}$ must be defined to maintain continuity as $\operatorname{Im}(\omega) \rightarrow 0$. This can be achieved by using a square root with a branch cut along the negative imaginary axis, that is, if $\xi=|\xi| e^{i \theta}$ for $-\pi / 2<\theta \leq 3 \pi / 2$, then $\sqrt{\xi}=\sqrt{|\xi|} e^{i \theta / 2}$. For a resonant mode above the light line, i.e. $\operatorname{Re}(k)>|\beta|, \alpha_{0}$ has a negative imaginary part, and the field blows up as $|x| \rightarrow \infty$.

\section{STRUCTURAL PERTURBATION}

In this section, we analyze the effects of structural perturbations to ASWs on 2D periodic structures with a reflection symmetry in the periodic direction. Let $u_{*}$ be a non-degenerate ASW with a positive frequency $\omega_{*}$ on a periodic structure given by a dielectric function $\epsilon_{*}$, where $\epsilon_{*}$ is even in $y$ and $u_{*}$ is odd in $y$. We assume $k_{*}=\omega_{*} / c$ satisfies

$$
0<k_{*}<2 \pi / L
$$

and consider a perturbed structure given by

$$
\epsilon(x, y)=\epsilon_{*}(x, y)+\delta F(x, y)
$$

where $\delta$ is small real parameter, and $F$ is the real perturbation profile satisfying $F(x, y)=0$ for $|x|>D$ and $\max |F|=1$. If $F$ is even in $y$, the perturbation can only shift the frequency on the real line, thus the perturbed structure still has an ASW near the original one [35]. In the following, we assume $F$ is non-symmetric, i.e., not an even function of $y$. 
We are interested in the complex frequency $\omega$ and the $Q$-factor of a nearby resonant mode $u$ with Bloch wavenumber $\beta=0$ on the perturbed structure. In the perturbation analysis, we expand $u$ and $\omega$ as

$$
\begin{aligned}
& u=u_{*}+u_{1} \delta+u_{2} \delta^{2}+u_{3} \delta^{3}+u_{4} \delta^{4}+\ldots \\
& \omega=\omega_{*}+\omega_{1} \delta+\omega_{2} \delta^{2}+\omega_{3} \delta^{3}+\omega_{4} \delta^{4}+\ldots
\end{aligned}
$$

Inserting Eqs. (7)-(8) into Eq. (1), and comparing terms of equal power in $\delta$, we obtain

$$
\begin{aligned}
\mathcal{L} u_{*}= & 0, \\
\mathcal{L} u_{1}= & -\left(2 k_{*} k_{1} \epsilon_{*}+k_{*}^{2} F\right) u_{*}, \\
\mathcal{L} u_{2}= & -\left(2 k_{*} k_{1} \epsilon_{*}+k_{*}^{2} F\right) u_{1} \\
& -\left(2 k_{*} k_{2} \epsilon_{*}+k_{1}^{2} \epsilon_{*}+2 k_{*} k_{1} F\right) u_{*},
\end{aligned}
$$

where $k_{j}=\omega_{j} / c$ for $j \geq 1$, and

$$
\mathcal{L}=\partial_{x}^{2}+\partial_{y}^{2}+k_{*}^{2} \epsilon_{*} .
$$

In addition, $u_{j}$ must satisfy a proper outgoing radiation condition as $|x| \rightarrow \infty$ and a periodic condition in the $y$ direction.

Equation (9) is simply the governing Helmholtz equation of the ASW. The first order term $u_{1}$ satisfies the inhomogeneous Eq. (10) which is singular and has no solution, unless the right hand side is orthogonal to $u_{*}$. Let $\Omega$ be the infinite strip given by $|y|<L / 2$ and $-\infty<x<\infty$. Multiplying $\bar{u}_{*}$ (the complex conjugate of $u_{*}$ ) to both sides of Eq. 10 and integrating on domain $\Omega$, we obtain

$$
k_{1}=\frac{\omega_{1}}{c}=-\frac{k_{*} \int_{\Omega} F\left|u_{*}\right|^{2} d \boldsymbol{r}}{2 \int_{\Omega} \epsilon\left|u_{*}\right|^{2} d \boldsymbol{r}} .
$$

Since $F$ is real, it is clear that $k_{1}$ is also real.

For $k_{1}$ given above, Eq. (10) has a solution. Similar to the plane wave expansion (3), $u_{1}$ can be written down explicitly for $|x|>D$. Importantly, condition (5) implies that $u_{1}$ contains only a single outgoing plane wave as $x \rightarrow \pm \infty$, that is

$$
u_{1} \sim b_{0}^{ \pm} e^{ \pm i k_{*} x}, \quad x \rightarrow \pm \infty,
$$

where $b_{0}^{ \pm}$are unknown coefficients. A formula for $k_{2}$ can be derived from the solvability condition of Eq. (11). In particular, the imaginary part of $k_{2}$ has the following simple formula

$$
\operatorname{Im}\left(k_{2}\right)=\frac{\operatorname{Im}\left(\omega_{2}\right)}{c}=-\frac{L\left(\left|b_{0}^{+}\right|^{2}+\left|b_{0}^{-}\right|^{2}\right)}{2 \int_{\Omega} \epsilon\left|u_{*}\right|^{2} d \boldsymbol{r}} .
$$

A derivation of Eq. (15) is given in Appendix A.

Notice that if $u_{1}$ radiates power to $x= \pm \infty, b_{0}^{+}$and $b_{0}^{-}$are nonzero, then $\operatorname{Im}\left(\omega_{2}\right) \neq 0$. In that case, the imaginary part of the complex frequency satisfies

$$
\operatorname{Im}(\omega) \approx \operatorname{Im}\left(\omega_{2}\right) \delta^{2}=-\frac{c L\left(\left|b_{0}^{+}\right|^{2}+\left|b_{0}^{-}\right|^{2}\right)}{2 \int_{\Omega} \epsilon\left|u_{*}\right|^{2} d \boldsymbol{r}} \delta^{2},
$$

and the $Q$-factor satisfies

$$
Q \approx \frac{k_{*} \int_{\Omega} \epsilon\left|u_{*}\right|^{2} d \boldsymbol{r}}{L\left(\left|b_{0}^{+}\right|^{2}+\left|b_{0}^{-}\right|^{2}\right)} \delta^{-2}
$$

If we can find a perturbation profile $F$ such that $b_{0}^{ \pm}=0$, then $\operatorname{Im}\left(\omega_{2}\right)=0$, and Eqs. (16) and (17) are no longer valid. In that case, $\operatorname{Im}\left(\omega_{3}\right)$ must also be zero, since otherwise, $\operatorname{Im}(\omega)$ changes signs when $\delta$ change signs. This is not possible, since $\operatorname{Im}(\omega)$ of a resonant mode is always negative. Therefore, if $u_{1}$ is non-radiative, we expect $\operatorname{Im}(\omega) \sim \delta^{4}$ and $Q \sim \delta^{-4}$. In the following, we derive a condition for $F$ that guarantees $b_{0}^{ \pm}=0$. This condition involves the ASW $u_{*}$ and related diffraction solutions for normal incident waves at the same $\omega_{*}$.

We consider two diffraction problems with normal incident waves $e^{i k_{*} x}$ and $e^{-i k_{*} x}$ given in the left and right homogeneous media, respectively. The solutions of these two diffraction problems are denoted as $v_{1}$ and $v_{2}$, respectively. Note that $v_{1}$ and $v_{2}$ can be chosen as even functions of $y$. The existence of a BIC implies that the corresponding diffraction problems have no uniqueness [4, 5], but the diffraction solutions are uniquely defined in the far field as $|x| \rightarrow \infty$. In fact, $v_{1}$ and $v_{2}$ have the following asymptotic formulae

$$
\begin{aligned}
& v_{1} \sim \begin{cases}e^{i k_{*} x}+S_{11} e^{-i k_{*} x}, & x \rightarrow-\infty \\
S_{21} e^{i k_{*} x}, & x \rightarrow+\infty,\end{cases} \\
& v_{2} \sim \begin{cases}S_{12} e^{-i k_{*} x}, & x \rightarrow-\infty \\
e^{-i k_{*} x}+S_{22} e^{i k_{*} x}, & x \rightarrow+\infty,\end{cases}
\end{aligned}
$$

where $S_{11}, S_{21}, S_{22}$ and $S_{12}$ are the reflection and transmission coefficients associated with the left and right incident waves, respectively, and they are the entries of a $2 \times 2$ scattering matrix $\boldsymbol{S}$. Due to the reciprocity and energy conservation, $\boldsymbol{S}$ is a symmetric and unitary matrix [49.

For Eq. 100, we would like to multiply both sides by $\bar{v}_{m}$ (for $m=1,2$ ) and integrate on domain $\Omega$. Since $u_{*}$ decays to zero exponentially as $x \rightarrow \pm \infty$, the right hand side, i.e.,

$$
G_{m}=-\int_{\Omega}\left(2 k_{*} k_{1} \epsilon_{*}+k_{*}^{2} F\right) u_{*} \bar{v}_{m} d \boldsymbol{r}
$$

is well defined. Since $\epsilon_{*}$ and $v_{m}$ are even in $y$, and $u_{*}$ is odd in $y, G_{m}$ can be simplified as

$$
G_{m}=-k_{*}^{2} \int_{\Omega} F u_{*} \bar{v}_{m} d \boldsymbol{r} .
$$

On the other hand, $v_{m}$ and $u_{1}$ (in general) do not decay to zero as $|x| \rightarrow \infty$, it is not immediately clear whether the right hand side can be integrated on the unbounded domain $\Omega$. However, for any $h \geq D$, we can define a rectangular domain $\Omega_{h}$ given by $|y|<L / 2$ and $|x|<h$, and evaluate the integral on $\Omega_{h}$, then take the limit as $h \rightarrow \infty$. Clearly, the limit must exist and

$$
\lim _{h \rightarrow \infty} \int_{\Omega_{h}} \bar{v}_{m} \mathcal{L} u_{1} d \boldsymbol{r}=G_{m}, \quad m=1,2 .
$$


In Appendix B, we show that

$$
\lim _{h \rightarrow \infty} \int_{\Omega_{h}} \bar{v}_{m} \mathcal{L} u_{1} d \boldsymbol{r}=2 i k_{*} L\left(b_{0}^{-} \bar{S}_{1 m}+b_{0}^{+} \bar{S}_{2 m}\right)
$$

Therefore,

$$
G_{m}=2 i k_{*} L\left(b_{0}^{-} \bar{S}_{1 m}+b_{0}^{+} \bar{S}_{2 m}\right), \quad m=1,2 .
$$

Since the scattering matrix $\boldsymbol{S}$ is invertible, it is clear that $b_{0}^{+}=b_{0}^{-}=0$ if and only if $G_{1}=G_{2}=0$. Therefore, if we choose a perturbation profile $F$ such that $G_{1}=G_{2}=$ 0 , then the $Q$-factors of resonant modes near the ASW should satisfy at least an inverse fourth order asymptotic relation, i.e. $Q \sim \delta^{-4}$. Notice that the condition $G_{1}=$ $G_{2}=0$ is unchanged, if we replace $v_{1}$ and $v_{2}$ in Eq. 20 by their linear combinations $w_{1}$ and $w_{2}$, as far as $w_{1}$ and $w_{2}$ are linearly independent. In Appendix D, we show that $w_{1}$ and $w_{2}$ can be chosen as real functions. Therefore, $G_{1}=G_{2}=0$ gives only two independent real conditions.

Many different perturbation profiles can satisfy the condition $G_{1}=G_{2}=0$. In the following, we show that if $G_{1}=G_{2}=0$ and $F$ is an odd function of $y$, then the $Q$-factors satisfy an inverse sixth order asymptotic relation, i.e. $Q \sim \delta^{-6}$. From the assumptions, it is clear that $k_{1}=0, b_{0}^{ \pm}=0, u_{1} \rightarrow 0$ as $|x| \rightarrow \infty$, and $k_{2}$ is real. Since the right hand side of Eq. 10 is an even function of $y$, we can choose $u_{1}$ as an even function of $y$. The right hand side of Eq. 11 is an odd function of $y$, so $u_{2}$ can be chosen as an odd function of $y$. Similar to Eq. (14, $u_{2}$ has the asymptotic formulae

$$
u_{2} \sim b_{1}^{ \pm} e^{ \pm k_{*} x}, \quad x \rightarrow \pm \infty
$$

where $b_{1}^{ \pm}$are unknown coefficients. The solvability of Eq. 44 for $u_{3}$ leads to $k_{3}=0$ as shown in Appendix C. In principle, $k_{4}$ can be obtained from the solvability of Eq. 45 for $u_{4}$. In Appendix C, we show that

$$
\operatorname{Im}\left(k_{4}\right)=\frac{\operatorname{Im}\left(\omega_{4}\right)}{c}=-\frac{L\left(\left|b_{1}^{+}\right|^{2}+\left|b_{1}^{-}\right|^{2}\right)}{2 \int_{\Omega} \epsilon\left|u_{*}\right|^{2} d \boldsymbol{r}} .
$$

Since $k_{1}=0$, the right hand side of Eq. 11 for $u_{2}$ is simplified to $-k_{*}^{2} F u_{1}-2 k_{*} k_{2} \epsilon_{*} u_{*}$. It decays to zero exponentially as $|x| \rightarrow \infty$, and is an odd function of $y$. Since the diffraction solutions $v_{m}$ (for $\left.m=1,2\right)$ are even in $y$, we have

$$
H_{m}=-\int_{\Omega}\left(k_{*}^{2} F u_{1}+2 k_{*} k_{2} \epsilon_{*} u_{*}\right) \bar{v}_{m} d \boldsymbol{r}=0 .
$$

Similar to Eq. 22, we multiply the left hand side of Eq. (11) by $\bar{v}_{m}$, integrate on domain $\Omega_{h}$, take the limit as $h \rightarrow \infty$, and obtain

$$
H_{m}=2 i k_{*} L\left(b_{1}^{-} \bar{S}_{1 m}+b_{1}^{+} \bar{S}_{2 m}\right), \quad m=1,2 .
$$

From Eqs. 25 and 26 above, we obtain $b_{1}^{-}=b_{1}^{+}=$ 0 . Therefore, $\operatorname{Im}\left(\omega_{4}\right)=0$ and $u_{2}$ does not radiate out power as $x \rightarrow \pm \infty$. Meanwhile, $\operatorname{Im}\left(\omega_{5}\right)$ must be zero, since otherwise $\operatorname{Im}(\omega)$ will have a wrong sign as $\delta$ changes signs. Since $k_{3}=0$, the right hand side of Eq. (44) for $u_{3}$ is even in $y$. It is possible for $u_{3}$ to radiate out power as $x \rightarrow \pm \infty$, then $\operatorname{Im}\left(\omega_{6}\right)$ is nonzero, $\operatorname{Im}(\omega) \sim \delta^{6}$, and $Q \sim \delta^{-6}$.

In summary, for a general real perturbation profile $F$, $Q$ is typically $O\left(1 / \delta^{2}\right)$ and is given in Eq. (17); if $F$ satisfies the condition $G_{1}=G_{2}=0$ for $G_{m}$ given in Eq. 20, then $Q$ is at least $O\left(1 / \delta^{4}\right)$; and if $F$ is odd in $y$ and $G_{1}=G_{2}=0$, then $Q$ is at least $O\left(1 / \delta^{6}\right)$.

\section{WAVENUMBER PERTURBATION}

A BIC with a Bloch wavenumber $\beta_{*}$ on a periodic structure is surrounded by resonant modes of different Bloch wavenumbers. The $Q$-factor of a nearby resonant mode with Bloch wavenumber $\beta$ is typically $O(1 / \mid \beta-$ $\left.\left.\beta_{*}\right|^{2}\right)$. It has been shown that if the BIC satisfies a special condition, then the $Q$-factor is at least $O\left(1 /\left|\beta-\beta_{*}\right|^{4}\right)$ 45. That condition was derived for BICs with or without symmetry protection, and it was automatically satisfied by symmetric standing waves which are BICs without symmetry protection [43. In this section, we study ASWs satisfying that special condition, and show that the $Q$-factors of the nearby resonant modes are typically $O\left(1 / \beta^{6}\right)$.

As in the previous section, we assume the periodic structure given by a dielectric function $\epsilon_{*}$ has a nondegenerate $\mathrm{ASW} u_{*}$, where $\epsilon_{*}$ is even in $y, u_{*}$ is odd in $y$, and the frequency $\omega_{*}$ of the ASW satisfies condition (5). For $\beta$ near $\beta_{*}=0$, the resonant mode $u$, given as a Bloch mode in Eq. 2 for a periodic function $\phi$, has a complex frequency $\omega$. Assuming $|\beta L|$ is small, we expand $\phi$ and $\omega$ in power series of $\beta$ :

$$
\begin{aligned}
& \omega=\omega_{*}+\omega_{1} \beta+\omega_{2} \beta^{2}+\omega_{3} \beta^{3}+\omega_{4} \beta^{4}+\ldots, \\
& \phi=u_{*}+\phi_{1} \beta+\phi_{2} \beta^{2}+\phi_{3} \beta^{3}+\phi_{4} \beta^{4}+\ldots,
\end{aligned}
$$

where $\phi_{j}$ for $j \geq 1$ are periodic in $y$ and must satisfy proper outgoing radiation conditions as $|x| \rightarrow \infty$. In terms of $\phi$, the Helmholtz equation becomes

$$
\frac{\partial^{2} \phi}{\partial x^{2}}+\frac{\partial^{2} \phi}{\partial y^{2}}+2 i \beta \frac{\partial \phi}{\partial y}+\left(k^{2} \epsilon_{*}-\beta^{2}\right) \phi=0 .
$$

Inserting Eqs. 27)-28 into Eq. 29, and comparing terms of equal power in $\delta$, we obtain

$$
\begin{aligned}
& \mathcal{L} u_{*}=0, \\
& \mathcal{L} \phi_{1}=-2 i \partial_{y} u_{*}-2 k_{*} k_{1} \epsilon_{*} u_{*},
\end{aligned}
$$

where $\mathcal{L}$ is the operator defined in Eq. 12 , and $k_{j}=$ $\omega_{j} / c$ for $j \geq 1$. The equations for $\phi_{2}, \phi_{3}$ and $\phi_{4}$ are also needed, and they will be given in simplified forms later in this section.

We can determine $k_{1}$ such that Eq. (31) has a solution. The result is

$$
k_{1}=\frac{\omega_{1}}{c}=\frac{-i \int_{\Omega} \bar{u}_{*} \partial_{y} u_{*} d \boldsymbol{r}}{k_{*} \int_{\Omega} \epsilon_{*}\left|u_{*}\right|^{2} d \boldsymbol{r}}=0 .
$$


Notice that $\bar{u}_{*} \partial_{y} u_{*}$ is odd in $y$, and thus its integral on $\Omega$ is zero. Since $\omega_{*}$ satisfies condition (5) and the medium for $|x|>D$ is homogeneous, $\phi_{1}$ has the asymptotic formulae

$$
\phi_{1} \sim d_{0}^{ \pm} e^{ \pm i k_{*} x}, \quad x \rightarrow \pm \infty,
$$

where $d_{0}^{ \pm}$are unknown coefficients. Meanwhile, since $k_{1}=0$, the equation for $\phi_{2}$ can be written as

$$
\mathcal{L} \phi_{2}=-2 i \partial_{y} \phi_{1}+\left(1-2 k_{*} k_{2} \epsilon_{*}\right) u_{*} .
$$

The solvability of Eq. (34) allows us to determine $k_{2}$. Following a procedure similar to that given in Appendix A, we obtain

$$
\operatorname{Im}\left(k_{2}\right)=\frac{\operatorname{Im}\left(\omega_{2}\right)}{c}=-\frac{L\left(\left|d_{0}^{+}\right|^{2}+\left|d_{0}^{-}\right|^{2}\right)}{2 \int_{\Omega} \epsilon_{*}\left|u_{*}\right|^{2} d \boldsymbol{r}} .
$$

Therefore, the $Q$-factors in general satisfy $Q \sim \beta^{-2}$. Multiplying the diffraction solutions $v_{1}$ and $v_{2}$ to Eq. (31) and integrating on $\Omega$, we obtain

$$
K_{m}=i k_{*} L\left(d_{0}^{-} \bar{S}_{1 m}+d_{0}^{+} \bar{S}_{2 m}\right),
$$

where

$$
K_{m}=-i \int_{\Omega} \bar{v}_{m} \partial_{y} u_{*} d \boldsymbol{r}, \quad m=1,2 .
$$

Clearly, the condition $d_{0}^{-}=d_{0}^{+}=0$ is equivalent to $K_{1}=$ $K_{2}=0$.

If $K_{1}=K_{2}=0$, then $d_{0}^{ \pm}=0$ and $\phi_{1}$ decays to zero exponentially as $|x| \rightarrow \infty$. Since $k_{1}=0$, the right hand side of Eq. (31) is even in $y$, thus $\phi_{1}$ can be chosen as an even function of $y$. The right hand side of Eq. (34) is odd in $y$, thus $\phi_{2}$ can be chosen as an odd function of $y$. Since there is only one opening diffraction channel, $\phi_{2}$ has the asymptotic formulae

$$
\phi_{2} \sim d_{1}^{ \pm} e^{ \pm i k_{*} x}, \quad x \rightarrow \pm \infty
$$

for unknown coefficients $d_{1}^{ \pm}$. However, as in the previous section, due to the symmetry mismatch between $\phi_{2}$ and plane waves $e^{ \pm i k_{*} x}$, we can show that $d_{1}^{-}=d_{1}^{+}=0$.

With $k_{1}=0$, the equation for $\phi_{3}$ becomes

$$
\mathcal{L} \phi_{3}=-2 i \partial_{y} \phi_{2}+\left(1-2 k_{*} k_{2} \epsilon_{*}\right) \phi_{1}-2 k_{*} k_{3} \epsilon_{*} \phi_{*} .
$$

It is easy to show that the solvability of $\phi_{3}$ gives rise to $k_{3}=0$. Since $k_{1}=k_{3}=0$, the equation for $\phi_{4}$ becomes

$$
\mathcal{L} \phi_{4}=-2 i \partial_{y} \phi_{3}+\left(1-2 k_{*} k_{2} \epsilon_{*}\right) \phi_{2}-\left(2 k_{*} k_{4}+k_{2}^{2}\right) \epsilon_{*} \phi_{*} .
$$

Following a procedure similar to that in Appendix C, we can show that

$$
\operatorname{Im}\left(k_{4}\right)=\frac{\operatorname{Im}\left(\omega_{4}\right)}{c}=-\frac{L\left(\left|d_{1}^{+}\right|^{2}+\left|d_{1}^{-}\right|^{2}\right)}{2 \int_{\Omega} \epsilon_{*}\left|u_{*}\right|^{2} d \boldsymbol{r}}=0 .
$$

Furthermore, $\operatorname{Im}\left(\omega_{5}\right)$ must be zero, since otherwise $\operatorname{Im}(\omega)$ can have the wrong sign when $\beta$ changes signs. Note that the right hand side of Eq. 39 is an even function of $y$, therefore, $\phi_{3}$ may radiate out power as $x \rightarrow \pm \infty$, we expect $\operatorname{Im}\left(\omega_{6}\right)$ to be nonzero, $\operatorname{Im}(\omega) \sim \beta^{6}$ and $Q \sim \beta^{-6}$.

\section{NUMERICAL EXAMPLES}

To validate and illustrate the theoretical results developed in the previous sections, we present numerical examples for periodic structures consisting of three arrays of circular dielectric cylinders surrounded by air. As shown in Fig. 11, the arrays are periodic in the $y$ direc-

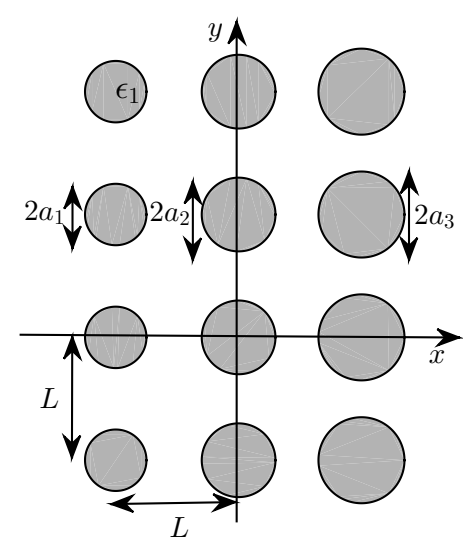

FIG. 1. A periodic structure (periodic in $y$ with period $L$ ) with three arrays of circular dielectric cylinders.

tion with a period $L$ and separated by a center-to-center distance $L$ in the $x$ direction. The radii of the cylinders in the three arrays are $a_{1}, a_{2}$ and $a_{3}$, respectively, and the dielectric constants of all cylinders are $\epsilon_{1}$. The coordinates are chosen so that the center of one cylinder is at the origin and the centers of the cylinders of the second array are on the $y$ axis. The domain $\Omega$ contains three cylinders.

To consider structural perturbations, we let $a_{1}=$ $0.29 L, a_{2}=0.3 L, a_{3}=0.31$ and $\epsilon_{1}=4$. For the $E$ polarization, the structure has three ASWs with normalized frequencies $\omega_{*} L /(2 \pi c)=0.6575,0.6715$ and 0.6862 , respectively, and they are referred to as ASW1, ASW2 and ASW3. The electric field patterns of these three ASWs shown in Fig. 2, For the first example, we consider a perturbation profile $F$ given by

$$
F(x, y)=\sum_{j=1}^{3} \eta_{j} F_{j}(x, y)
$$

where $\eta_{1}, \eta_{2}$ and $\eta_{3}$ are real constants, $F_{1}, F_{2}$ and $F_{3}$ are defined (on domain $\Omega$ ) by

$$
F_{j}(x, y)= \begin{cases}\sin \frac{\pi y}{2 a_{j}}, & \text { in the } j \text {-th cylinder } \\ 0, & \text { otherwise }\end{cases}
$$

for $j=1,2$ and 3 . We are interested in a profile $F$ such that $G_{1}=G_{2}=0$, i.e.

$$
\sum_{j=1}^{3} \eta_{j} \int_{\Omega} F_{j} u_{*} \bar{v}_{m} d \boldsymbol{r}=0, \quad m=1,2 .
$$



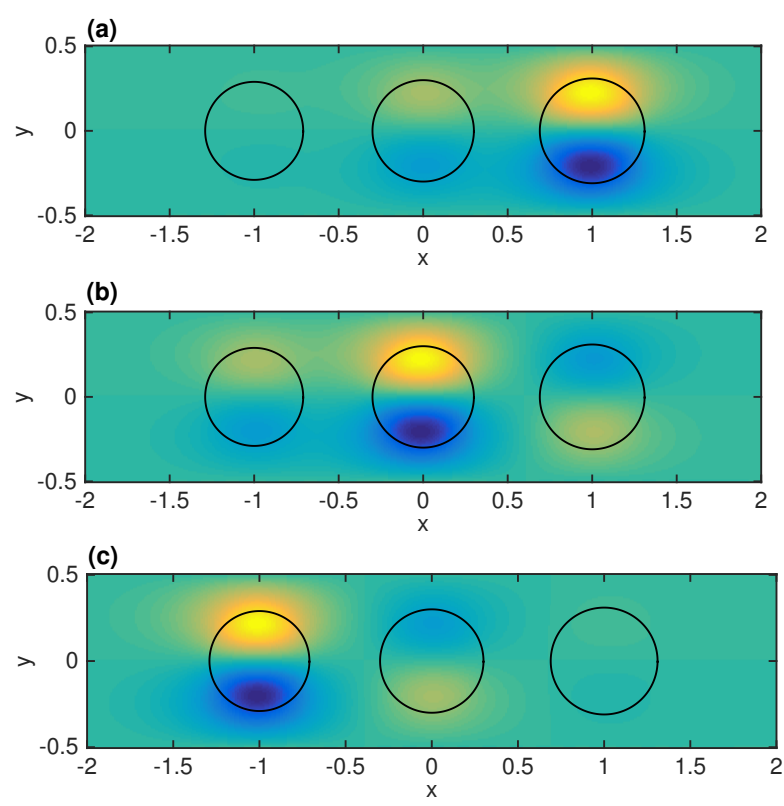

FIG. 2. Wave field patterns of antisymmetric standing waves on a periodic structure with three arrays of cylinders: (a) ASW1, (b) ASW2, (c) ASW3.

As we mentioned in Sec. III the condition $G_{1}=G_{2}=0$ is equivalent to two real independent conditions, thus, by fixing any one of $\eta_{1}, \eta_{2}$ and $\eta_{3}$, we can solve the other two from the above system. After that, we can scale $F$ such that $\max _{(x, y) \in \Omega}|F|=1$. The obtained values of $\eta_{1}, \eta_{2}$ and $\eta_{3}$, corresponding to the three ASWs on the unperturbed structures, are listed in Table I below.

\begin{tabular}{c|c||c|c|c}
\hline \hline BICs & $\omega_{*} L /(2 \pi c)$ & $\eta_{1}$ & $\eta_{2}$ & $\eta_{3}$ \\
\hline ASW1 & 0.6575 & 1 & -0.5077 & 0.1255 \\
\hline ASW2 & 0.6715 & 1 & -0.2380 & 0.2445 \\
\hline ASW3 & 0.6862 & 0.0844 & 0.4679 & 1 \\
\hline \hline
\end{tabular}

TABLE I. Example 1: coefficients of antisymmetric perturbation profiles $F$ satisfying the condition $G_{1}=G_{2}=0$.

Since $F_{1}, F_{2}$ and $F_{3}$ are odd in $y, F$ is also odd in $y$, thus the $Q$-factors of the resonant modes should satisfy $Q \sim \delta^{-6}$. In Fig. 3, we show the $Q$-factors of the resonant modes near the three ASWs for different values of $\delta$. The numerical results clearly confirm the inverse sixth order relation between $Q$ and $\delta$.

To calculate the $Q$-factors of the resonant modes, we solve an eigenvalue problem formulated using a mixed Fourier-Chebyshev pseudospectral method to discretize the three circular disks (corresponding to the cross section of the three cylinders in $\Omega$ ) [50, and cylindrical and plane wave expansions outside the cylinders [51.

For the second example, we consider perturbation profiles that are asymmetric in the $y$ direction. For the same unperturbed structure with three arrays of cylinders and the same $F_{2}$ and $F_{3}$ given in Eq. $\sqrt{42}$, we define a new

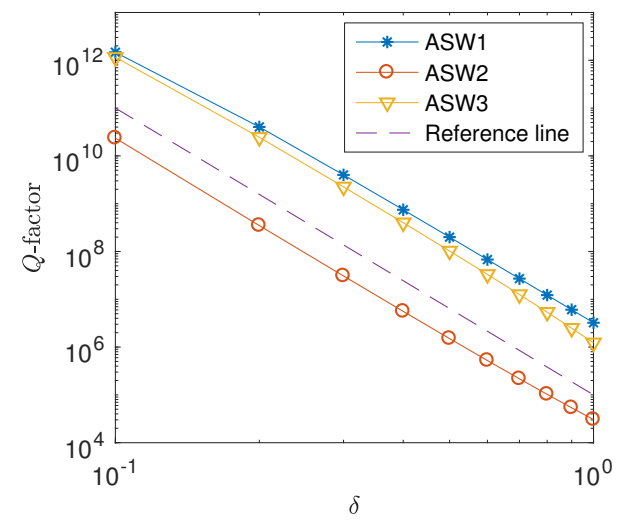

FIG. 3. Example 1: $Q$-factors of resonant modes near ASW1, ASW2 and ASW3 for perturbed structures with coefficients of $F$ listed in Table \. The dashed line is the reference line for $Q=10^{5} / \delta^{6}$.

$F_{1}$ (on domain $\Omega$ ) by

$$
F_{1}(x, y)= \begin{cases}\sin \left(\frac{\pi y}{2 a_{1}}+\frac{\pi}{4}\right), & \text { in the first cylinder } \\ 0, & \text { otherwise }\end{cases}
$$

For $F$ given in Eq. (41), we solve $\eta_{1}, \eta_{2}$ and $\eta_{3}$ such that $G_{1}=G_{2}=0$ for each ASW. The results are listed in Table II below. Since $F$ is neither even in $y$ nor odd in

\begin{tabular}{c|c||c|c|c}
\hline \hline BICs & $\omega_{*} L /(2 \pi c)$ & $\eta_{1}$ & $\eta_{2}$ & $\eta_{3}$ \\
\hline ASW1 & 0.6575 & 1 & -0.3583 & 0.0885 \\
\hline ASW2 & 0.6715 & 1 & -0.1683 & 0.1729 \\
\hline ASW3 & 0.6862 & 0.1194 & 0.4679 & 1 \\
\hline \hline
\end{tabular}

TABLE II. Example 2: coefficients of asymmetric perturbation profiles satisfying the condition $G_{1}=G_{2}=0$.

$y$, the $Q$-factors of nearby resonant modes should satisfy $Q \sim \delta^{-4}$. Numerical results for the $Q$-factors are shown in Fig. 3 in logarithmic scales, and they agree very well with the inverse fourth order relation between $Q$ and $\delta$.

The third example is designed to validate the perturbation results of Sec. IV] We consider the same periodic structure with three arrays of cylinders as shown in Fig. 1. keep $a_{2}=0.3 L$ and $\epsilon_{1}=4$ as in the first example, but assume $a_{1}=a_{3}$. First, we show that for $a_{1}=a_{3} \approx 0.33033 L$, the periodic structure has an ASW satisfying the condition $K_{1}=K_{2}=0$. As shown in Appendix $\mathrm{D}$, the diffraction solutions $v_{1}$ and $v_{2}$ have two linear combinations $w_{1}$ and $w_{2}$ that are real even functions of $y$. Since the periodic structure of this example is symmetric in $x, w_{1}$ and $w_{2}$ can be be written down explicitly as

$$
w_{1}=\frac{e^{-i \theta_{1}}}{\sqrt{2}}\left(v_{1}+v_{2}\right), \quad w_{2}=\frac{e^{-i \theta_{2}}}{\sqrt{2}}\left(v_{1}-v_{2}\right),
$$




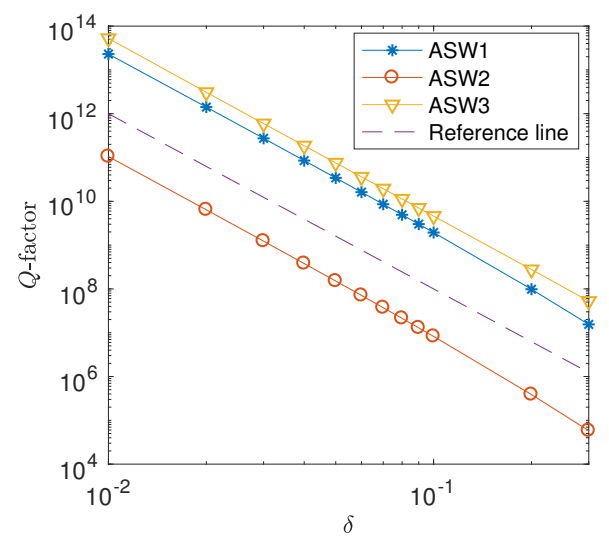

FIG. 4. Example 2: $Q$-factors of resonant modes near ASW1, ASW2 and ASW3 for perturbed structures with coefficients of $F$ listed in Table II] The dashed line is the reference line for $Q=10^{4} / \delta^{4}$.

where $\theta_{1}$ and $\theta_{2}$ satisfy $R+T=e^{2 i \theta_{1}}$ and $R-T=e^{2 i \theta_{2}}$, $R=S_{11}=S_{22}$ and $T=S_{12}=S_{21}$ are entries of the scattering matrix $\boldsymbol{S}$. Importantly, $w_{1}$ is even in $x$ and $w_{2}$ is odd in $x$. Clearly, the condition $K_{1}=K_{2}=0$ is equivalent to $\tilde{K}_{1}=\tilde{K}_{2}=0$, where

$$
\tilde{K}_{m}=\int_{\Omega} \bar{w}_{m} \partial_{y} u_{*} d \boldsymbol{r}, \quad m=1,2 .
$$

In Fig. 5 , we show $\tilde{K}_{1}$ and $\tilde{K}_{2}$ as functions of $a_{1}$ for

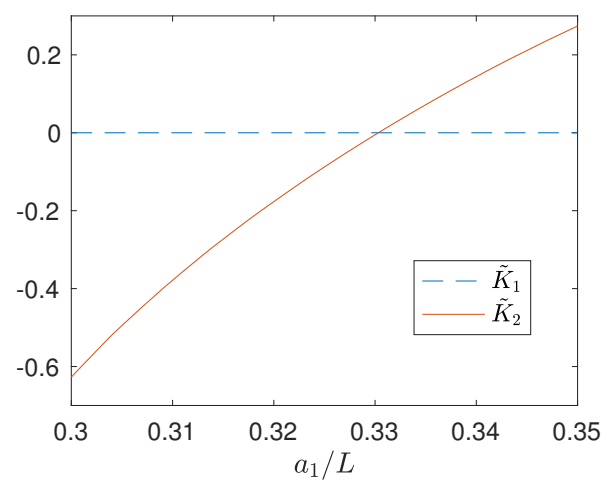

FIG. 5. Example 3: $\tilde{K}_{1}$ and $\tilde{K}_{2}$ [defined in Eq. 43 , with unit $L]$ as functions of $a_{1}$ for an ASW on a periodic structure with three arrays of cylinders with radii $a_{1}, a_{2}=0.3 L$ and $a_{3}=a_{1}$.

an ASW with a wave field pattern similar to that of ASW2 in Fig. 2(b). The ASW is normalized such that $\int_{\Omega}\left|u_{*}\right|^{2} d \boldsymbol{r} / L^{2} \stackrel{2}{=} 1$. Since the ASW is odd in $x$, we have $\tilde{K}_{1}=0$. From Fig. 5 it is clear that $\tilde{K}_{2}$ is real and changes signs. Therefore, $\tilde{K}_{2}$ must have a zero. Solving $\tilde{K}_{2}=0$ by a numerical method, we obtain $a_{1}=a_{3}=0.33033 L$. The normalized frequency of the corresponding ASW is $\omega_{*} L /(2 \pi c)=0.6361$. Its wave field pattern is shown in Fig. 6 .

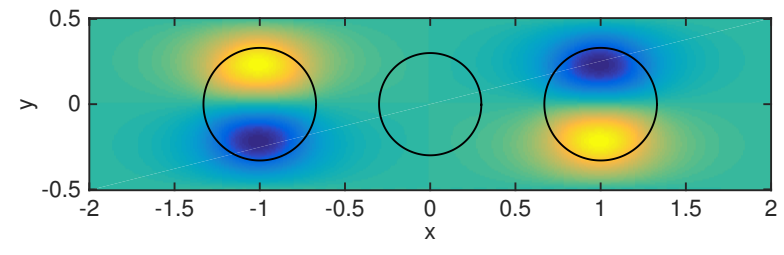

FIG. 6. Example 3: Wave field pattern of an ASW with frequency $\omega_{*} L /(2 \pi c)=0.6361$ on a periodic structure with $a_{1}=a_{3}=0.33033 L$ and $a_{2}=0.3 L$.

Finally, we calculate the $Q$-factors of the nearby resonant modes with $\beta$ close to 0 . The numerical results are shown in Fig. 7, and they agree well with the inverse

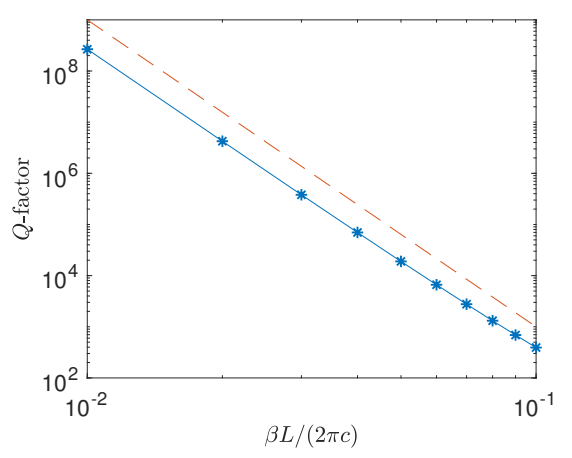

FIG. 7. Example 3: $Q$-factors of resonant modes near the ASW of Fig. 6. The dashed line is the reference line for $Q=$ $10^{-3}[2 \pi /(\beta L)]^{6}$.

sixth order relation between $Q$ and $\beta$.

\section{CONCLUSION}

The BICs are useful mainly because high- $Q$ resonances can be easily created by slightly changing the structure or other parameters. For theoretical understanding and practical applications, it is important to find out how the $Q$-factors of the resonant modes depend on the amplitude of the perturbations. Near a symmetry-protected BIC, a symmetry-breaking structural perturbation of amplitude $\delta$ typically creates a resonant mode with a $Q$-factor proportional to $1 / \delta^{2}$. For ASWs on $2 \mathrm{D}$ periodic structures with a reflection symmetry, we show that the $Q$-factor can be $O\left(1 / \delta^{4}\right)$ if the perturbation satisfies a simple condition, and it becomes $O\left(1 / \delta^{6}\right)$ if in addition to that condition, the perturbation is antisymmetric. On the other hand, a BIC on a periodic structure is always surrounded by resonant modes depending on the Bloch wave vector. It is well known that the $Q$-factors of these resonant modes tend to infinity as the wave vector tends to that of the BIC. For 2D periodic structures, we show that if an ASW satisfies a condition first derived in 45, the $Q$ factors of nearby resonant modes are $O\left(1 / \beta^{6}\right)$, where $\beta$ is 
the Bloch wavenumber. These theoretical results can be useful for practical applications where high- $Q$ resonances are needed. The $O\left(1 / \delta^{6}\right)$ result on structural perturbation implies that large $Q$-factors are possible without using a very small $\delta$, probably making the fabrication process somewhat easier. The $O\left(1 / \beta^{6}\right)$ result on wavenumber perturbation implies that strong resonances can be induced by incident waves with a wider range of incident angles.

The current study is limited to ASWs which are symmetry protected BICs. For BICs unprotected by symmetry, we have derived a condition on the perturbation profile $F$ such that the $Q$-factors of nearby resonant modes are proportional to $1 / \delta^{4}$, but have not found a condition for $Q \sim 1 / \delta^{6}$. Our study is also limited to $2 \mathrm{D}$ structures with a $1 \mathrm{D}$ periodicity. It is worthwhile to find similar results for three-dimensional biperiodic structures such as photonic crystal slabs. On actual fabricated structures, the $Q$-factors are limited by many practical issues, such as fabrication errors, material losses, finite sizes, and loss of periodicity (for periodic structures). It is necessary to have a comprehensive study on the different factors, but we still expect the structures satisfying the conditions for high- $Q$ resonances have advantages in realistic devices.

\section{ACKNOWLEDGMENTS}

The authors acknowledge support from the Natural Science Foundation of Chongqing, China (Grant No. cstc2019jcyj-msxmX0717), the Science and Technology Research Program of Chongqing Municipal Education Commission, China (Grant No. KJ1706155), and the Research Grants Council of Hong Kong Special Administrative Region, China (Grant No. CityU 11304117).

\section{APPENDIX A}

It is easy to verify that $\int_{\Omega} \bar{u}_{*} \mathcal{L} u_{2} d \boldsymbol{r}=0$. See Appendix in Ref. 45. Multiplying both sides of Eq. (11) and integrating on $\Omega$, we obtain

$$
k_{2}=\frac{\int_{\Omega} R d \boldsymbol{r}-\int_{\Omega}\left(k_{1}^{2} \epsilon_{*}+2 k_{*} k_{1} F\right)\left|u_{*}\right|^{2} d \boldsymbol{r}}{2 k_{*} \int_{\Omega} \epsilon_{*}\left|u_{*}\right|^{2} d \boldsymbol{r}},
$$

where $R=-\left(2 k_{*} k_{1} \epsilon_{*}+k_{*}^{2} F\right) \bar{u}_{*} u_{1}$. Therefore,

$$
\operatorname{Im}\left(k_{2}\right)=\frac{\operatorname{Im}\left[\int_{\Omega} R d \boldsymbol{r}\right]}{2 k_{*} \int_{\Omega} \epsilon\left|u_{*}\right|^{2} d \boldsymbol{r}} .
$$

From the complex conjugate of Eq. 10, we obtain

$$
\int_{\Omega_{h}} u_{1} \overline{\mathcal{L}} \bar{u}_{1} d \boldsymbol{r}=\int_{\Omega_{h}} R d \boldsymbol{r}
$$

where $\Omega_{h}$ is a rectangle given by $|y|<L / 2$ and $|x|<h$ for $h>D$. It is easy to verify that

$$
\int_{\Omega_{h}} u_{1} \overline{\mathcal{L}} \bar{u}_{1} d \boldsymbol{r}=\int_{\partial \Omega_{h}} u_{1} \frac{\partial \bar{u}_{1}}{\partial \nu} d s
$$

$$
+\int_{\Omega_{h}}\left(k_{*}^{2} \epsilon_{*}\left|u_{1}\right|^{2}-\left|\nabla u_{1}\right|^{2}\right) d \boldsymbol{r},
$$

where $\partial \Omega_{h}$ is the boundary of $\Omega_{h}$ and $\nu$ is its unit outward normal vector. The second term in the right hand side above is real. Therefore,

$$
\operatorname{Im}\left[\int_{\Omega_{h}} R d \boldsymbol{r}\right]=\operatorname{Im}\left[\int_{\partial \Omega_{h}} u_{1} \frac{\partial \bar{u}_{1}}{\partial \nu} d s\right] .
$$

Since $u_{1}$ is periodic in $y$, the line integrals at $y= \pm L / 2$ are canceled. Therefore

$$
\int_{\partial \Omega_{h}} u_{1} \frac{\partial \bar{u}_{1}}{\partial \nu} d s=\int_{-L / 2}^{L / 2}\left[u_{1} \frac{\partial \bar{u}_{1}}{\partial x}\right]_{x=-h}^{x=h} d y
$$

where $\left.P(x, y)\right|_{x=-h} ^{x=h}$ denotes $P(h, y)-P(-h, y)$.

For $|x|>D$, the equation for $u_{1}$ is quite simple. It is not difficult to see that

$$
u_{1}=b_{0}^{ \pm} e^{ \pm i k_{*} x}+\sum_{j \neq 0} f_{j}^{ \pm}(x) e^{i 2 \pi j y / L} e^{ \pm \gamma_{* j} x}
$$

for $x>D$ and $x<-D$ respectively, where $\gamma_{* j}=$ $-i \sqrt{k_{*}^{2}-(2 j \pi / L)^{2}}$ for $j \neq 0$ is positive, $b_{0}^{ \pm}$are unknown coefficients, and $f_{j}^{ \pm}(x)(j \neq 0)$ are unknown linear polynomials of $x$. The above gives

$$
\lim _{h \rightarrow+\infty} \int_{-L / 2}^{L / 2}\left[u_{1} \frac{\partial \bar{u}_{1}}{\partial x}\right]_{x=-h}^{x=h} d y=-i k_{*} L\left(\left|b_{0}^{+}\right|^{2}+\left|b_{0}^{-}\right|^{2}\right),
$$

and $\operatorname{Im}\left[\int_{\Omega} R d \boldsymbol{r}\right]=-L k_{*}\left(\left|b_{0}^{+}\right|^{2}+\left|b_{0}^{-}\right|^{2}\right)$, and finally Eq. (15).

\section{APPENDIX B}

To show Eq. 21], we notice that

$$
\bar{v}_{1} \mathcal{L} u_{1}-u_{1} \overline{\mathcal{L}} \bar{v}_{1}=\nabla \cdot\left[\bar{v}_{1} \nabla u_{1}-u_{1} \nabla \bar{v}_{1}\right] .
$$

Since $v_{1}$ satisfies the Helmholtz equation and both $u_{1}$ and $v_{1}$ are periodic in $y$, we have

$$
\int_{\Omega_{h}} \bar{v}_{1} \mathcal{L} u_{1} d \boldsymbol{r}=\int_{\partial \Omega_{h}}\left[\bar{v}_{1} \frac{\partial u_{1}}{\partial \nu}-u_{1} \frac{\partial \bar{v}_{1}}{\partial \nu}\right] d s .
$$

In the right hand side above, the integrals on the two edges at $y= \pm L / 2$ are canceled. Therefore

$$
\int_{\Omega_{h}} \bar{v}_{1} \mathcal{L} u_{1} d \boldsymbol{r}=\int_{-L / 2}^{L / 2}\left[\bar{v}_{1} \frac{\partial u_{1}}{\partial x}-u_{1} \frac{\partial \bar{v}_{1}}{\partial x}\right]_{x=-h}^{x=h} d y .
$$

Based on the asymptotic formula (18), it is easy to show that as $h \rightarrow+\infty$, the right hand side above tends to $2 i k_{*} L\left(b_{0}^{-} \bar{S}_{11}+b_{0}^{+} \bar{S}_{21}\right)$. This leads to Eq. 21) for $m=1$. The case for $m=2$ is similar. 


\section{APPENDIX C}

Inserting Eqs. (7)-(8) into Eq. (1), and comparing terms of equal power in $\delta$, we obtain the following equation for $u_{3}$

$$
\begin{aligned}
\mathcal{L} u_{3}= & -k_{*}^{2} F u_{2}-2 k_{*} k_{2} \epsilon_{*} u_{1} \\
& -\left(2 k_{*} k_{3} \epsilon_{*}+2 k_{*} k_{2} F\right) u_{*}
\end{aligned}
$$

In the above, the result $k_{1}=0$ is used. Multiplying both sides of Eq. (44) by $\bar{u}_{*}$ and integrating on $\Omega$, we have

$$
k_{3}=-\frac{\int_{\Omega} k_{*} F u_{2} \bar{u}_{*}+2 k_{2} \epsilon_{*} u_{1} \bar{u}_{*}+2 k_{2} F\left|u_{*}\right|^{2} d \mathbf{r}}{2 \int_{\Omega} \epsilon_{*}\left|u_{*}\right|^{2} d \mathbf{r}} .
$$

Note that $F, u_{*}$ and $u_{2}$ are odd functions of $y$, and $\epsilon_{*}$ and $u_{1}$ are even functions of $y$, thus $k_{3}=0$.

The governing equation for $u_{4}$ is

$$
\begin{aligned}
\mathcal{L} u_{4}= & -k_{*}^{2} F u_{3}-2 k_{*} k_{2} \epsilon_{*} u_{2}-2 k_{*} k_{2} F u_{1} \\
& -\left(2 k_{*} k_{4} \epsilon_{*}+k_{2}^{2} \epsilon_{*}\right) u_{*} .
\end{aligned}
$$

The results $k_{1}=k_{3}=0$ have been used to simplify the above equation. Multiplying both sides of Eq. 45 and integrating on $\Omega$, we obtain

$$
k_{4}=\frac{\int_{\Omega} W d \boldsymbol{r}-k_{2}^{2} \int_{\Omega} \epsilon_{*}\left|u_{*}\right|^{2} d \boldsymbol{r}}{2 k_{*} \int_{\Omega} \epsilon_{*}\left|u_{*}\right|^{2} d \boldsymbol{r}}
$$

where

$$
W=-2 k_{*} k_{2} F \bar{u}_{*} u_{1}-2 k_{*} k_{2} \epsilon_{*} \bar{u}_{*} u_{2}-k_{*}^{2} F \bar{u}_{*} u_{3} .
$$

Note that $k_{2}$ is real. Therefore,

$$
\operatorname{Im}\left(k_{4}\right)=\frac{\operatorname{Im}\left[\int_{\Omega} W d \boldsymbol{r}\right]}{2 k_{*} \int_{\Omega} \epsilon\left|u_{*}\right|^{2} d \boldsymbol{r}} .
$$

From Eqs. (10), (11) and 44, we have

$\operatorname{Im}\left[\int_{\Omega_{h}}\left(u_{1} \overline{\mathcal{L}} \bar{u}_{3}-\bar{u}_{3} \mathcal{L} u_{1}+u_{2} \overline{\mathcal{L}} \bar{u}_{2}\right) d \boldsymbol{r}\right]=\operatorname{Im}\left[\int_{\Omega_{h}} W d \boldsymbol{r}\right]$.

Since $u_{1}$ decays to zero exponentially, $\int_{\Omega} \bar{u}_{3} \mathcal{L} u_{1} d \boldsymbol{r}$ and $\int_{\Omega} u_{1} \overline{\mathcal{L}} \bar{u}_{3} d \boldsymbol{r}$ are well defined. It is easy to show that $\int_{\Omega}\left(u_{1} \overline{\mathcal{L}} \bar{u}_{3}-\bar{u}_{3} \mathcal{L} u_{1}\right) d \boldsymbol{r}=0$. On the other hand, due to the asymptotic formula $(23)$, we have

$$
\lim _{h \rightarrow \infty} \int_{\Omega_{h}} u_{2} \overline{\mathcal{L}} \bar{u}_{2} d \boldsymbol{r}=-i k_{*} L\left(\left|b_{1}^{-}\right|^{2}+\left|b_{1}^{+}\right|^{2}\right) .
$$

Therefore,

$$
\begin{aligned}
& \lim _{h \rightarrow \infty} \int_{\Omega_{h}}\left(u_{1} \overline{\mathcal{L}} \bar{u}_{3}-\bar{u}_{3} \mathcal{L} u_{1}+u_{2} \overline{\mathcal{L}} \bar{u}_{2}\right) d \boldsymbol{r} \\
& =-i L k_{*}\left(\left|b_{1}^{-}\right|^{2}+\left|b_{1}^{+}\right|^{2}\right) .
\end{aligned}
$$

That means

$$
\operatorname{Im}\left[\int_{\Omega} W d \boldsymbol{r}\right]=-L k_{*}\left(\left|b_{1}^{-}\right|^{2}+\left|b_{1}^{+}\right|^{2}\right) .
$$

This leads to Eq. 24.

\section{APPENDIX D}

The $2 \times 2$ scattering matrix $\boldsymbol{S}$ is symmetric and unitary due to reciprocity and conservation of energy. Therefore, it has two real orthonormal eigenvectors $\boldsymbol{a}_{1}$ and $\boldsymbol{a}_{2}$. The corresponding eigenvalues $\lambda_{1}$ and $\lambda_{2}$ have unit magnitudes, i.e., for $j=1$ and $2, \lambda_{j}=\exp \left(i 2 \theta_{j}\right)$ where $\theta_{j}$ is real. Writing down the eigenvectors as

$$
\mathbf{a}_{1}=\left[\begin{array}{l}
a_{11} \\
a_{21}
\end{array}\right], \quad \mathbf{a}_{2}=\left[\begin{array}{l}
a_{12} \\
a_{22}
\end{array}\right]
$$

we define two functions $w_{1}$ and $w_{2}$ by

$$
w_{j}=e^{-i \theta_{j}}\left(a_{1 j} v_{1}+a_{2 j} v_{2}\right), \quad j=1,2 .
$$

then

$$
w_{j} \sim \begin{cases}2 a_{1 j} \cos \left(k_{*} x-\theta_{j}\right), & x \rightarrow-\infty \\ 2 a_{2 j} \cos \left(k_{*} x+\theta_{j}\right), & x \rightarrow+\infty\end{cases}
$$

Since $w_{j}$ and $\operatorname{Re}\left(w_{j}\right)$ satisfy the same Helmholtz equation and the same asymptotic conditions for $x \rightarrow \pm \infty$, we can replace $w_{j}$ by $\operatorname{Re}\left(w_{j}\right)$.

If the structure is symmetric in $x$, then the reflection coefficients for left and right incident waves are the same, thus

$$
\boldsymbol{S}=\left[\begin{array}{ll}
R & T \\
T & R
\end{array}\right]
$$

Then $\lambda_{1}=R+T, \lambda_{2}=R-T$, and

$$
\mathbf{a}_{1}=\frac{1}{\sqrt{2}}\left[\begin{array}{l}
1 \\
1
\end{array}\right], \quad \mathbf{a}_{2}=\frac{1}{\sqrt{2}}\left[\begin{array}{c}
1 \\
-1
\end{array}\right] .
$$

[1] J. von Neumann and E. Wigner, "Über merkwürdige diskrete eigenwerte," Z. Physik 50, 291-293 (1929).

[2] C. W. Hsu, B. Zhen, A. D. Stone, J. D. Joannopoulos, and M. Soljačić, "Bound states in the continuum," Nat. Rev. Mater. 1, 16048 (2016).

[3] K. Koshelev, G. Favraud, A. Bogdanov, Y. Kivshar, and A. Fratalocchi, "Nonradiating photonics with res- onant dielectric nanostructures," Nanophotonics 8, 725745 (2019)

[4] A.-S. Bonnet-Bendhia and F. Starling, "Guided waves by electromagnetic gratings and nonuniqueness examples for the diffraction problem," Math. Methods Appl. Sci. 17, 305-338 (1994).

[5] S. P. Shipman and S. Venakides, "Resonance and bound 
states in photonic crystal slabs," SIAM J. Appl. Math. 64, 322-342 (2003).

[6] D. V. Evans, M. Levitin and D. Vassiliev, "Existence theorems for trapped modes," J. Fluid Mech. 261, 21-31 (1994).

[7] E. N. Bulgakov and A. F. Sadreev, "Bound states in the continuum in photonic waveguides inspired by defects," Phys. Rev. B 78, 075105 (2008).

[8] Y. Plotnik, O. Peleg, F. Dreisow, M. Heinrich, S. Nolte, A. Szameit, and M. Segev, "Experimental observation of optical bound states in the continuum," Phys. Rev. Lett. 107, 183901 (2011).

[9] S. Weimann, Y. Xu, R. Keil, A. E. Miroshnichenko, A. Tünnermann, S. Nolte, A. A. Sukhorukov, A. Szameit, and Y. S. Kivshar, "Compact surface Fano states embedded in the continuum of the waveguide arrays," Phys. Rev. Lett. 111, 240403 (2013).

[10] C. L. Zou, J.-M. Cui, F.-W. Sun, X. Xiong, X.-B. Zou, Z.F. Han, and G.-C. Guo, "Guiding light through optical bound states in the continuum for ultrahigh-Q microresonantors," Laser \& Photonics Rev. 9, 114-119 (2015).

[11] J. Gomis-Bresco, D. Artigas, and L. Tprner, "Anisotropy-induced photonic bound states in the continuum," Nature Photonics 11, 232-237 (2017)

[12] P. Paddon and J. F. Young, "Two-dimensional vectorcoupled-mode theory for textured planar waveguides," Phys. Rev. B 61, 2090-2101 (2000).

[13] S. G. Tikhodeev, A. L. Yablonskii, E. A Muljarov, N. A. Gippius, and T. Ishihara, "Quasi-guided modes and optical properties of photonic crystal slabs," Phys. Rev. B 66, 045102 (2002).

[14] S. Shipman and D. Volkov, "Guided modes in periodic slabs: existence and nonexistence," SIAM J. Appl. Math. 67, 687-713 (2007).

[15] J. Lee, B. Zhen, S. L. Chua, W. Qiu, J. D. Joannopoulos, M. Soljačić, and O. Shapira, "Observation and differentiation of unique high-Q optical resonances near zero wave vector in macroscopic photonic crystal slabs," Phys. Rev. Lett. 109, 067401 (2012).

[16] Z. Hu and Y. Y. Lu, "Standing waves on two-dimensional periodic dielectric waveguides," Journal of Optics 17, 065601 (2015).

[17] R. Porter and D. Evans, "Embedded Rayleigh-Bloch surface waves along periodic rectangular arrays," Wave Motion 43, 29-50 (2005).

[18] D. C. Marinica, A. G. Borisov, and S. V. Shabanov, "Bound states in the continuum in photonics," Phys. Rev. Lett. 100, 183902 (2008).

[19] C. W. Hsu, B. Zhen, S.-L. Chua, S. G. Johnson, J. D. Joannopoulos, and M. Soljačić, "Bloch surface eigenstates within the radiation continuum," Light Sci. Appl. 2, e84 (2013).

[20] C. W. Hsu, B. Zhen, J. Lee, S.-L. Chua, S. G. Johnson, J. D. Joannopoulos, and M. Soljačić, "Observation of trapped light within the radiation continuum," Nature 499, 188-191 (2013).

[21] Y. Yang, C. Peng, Y. Liang, Z. Li, and S. Noda, "Analytical perspective for bound states in the continuum in photonic crystal slabs," Phys. Rev. Lett. 113, 037401 (2014).

[22] E. N. Bulgakov and A. F. Sadreev, "Bloch bound states in the radiation continuum in a periodic array of dielectric rods," Phys. Rev. A 90, 053801 (2014).

[23] E. N. Bulgakov and A. F. Sadreev, "Light trapping above the light cone in one-dimensional array of dielectric spheres," Phys. Rev. A 92, 023816 (2015).

[24] R. Gansch, S. Kalchmair, P. Genevet, T. Zederbauer, H. Detz, A. M. Andrews, W. Schrenk, F. Capasso, M. Lončar, and G. Strasser, "Measurement of bound states in the continuum by a detector embedded in a photonic crystal," Light: Science \& Applications 5, e16147 (2016).

[25] L. Li and H. Yin, "Bound States in the Continuum in double layer structures," Sci. Rep. 6, 26988 (2016).

[26] X. Gao, C. W. Hsu, B. Zhen, X. Lin, J. D. Joannopoulos, M. Soljačić, and H. Chen, "Formation mechanism of guided resonances and bound states in the continuum in photonic crystal slabs," Sci. Rep. 6, 31908 (2016).

[27] L. Ni, Z. Wang, C. Peng, and Z. Li, "Tunable optical bound states in the continuum beyond in-plane symmetry protection," Phys. Rev. B 94, 245148 (2016).

[28] L. Yuan and Y. Y. Lu, "Propagating Bloch modes above the lightline on a periodic array of cylinders," J. Phys. B: Atomic, Mol. and Opt. Phys. 50, 05LT01 (2017).

[29] E. N. Bulgakov and A. F. Sadreev, "Bound states in the continuum with high orbital angular momentum in a dielectric rod with periodically modulated permittivity," Phys. Rev. A 96, 013841 (2017).

[30] Z. Hu and Y. Y. Lu, "Propagating bound states in the continuum at the surface of a photonic crystal," J. Opt. Soc. Am. B 34, 1878-1883 (2017).

[31] S. Dai, L. Liu, D. Han, and J. Zi, "From topologically protected coherent perfect reflection to bound states in the continuum," Phys. Rev. B 98, 081405(R) (2018).

[32] Q. Song, M. Zhao, L. Liu, J. Chai, G. He, H. Xiang, D. Han, and J. Zi, "Observation of bound states in the continuum in the dimerized chain," Phys. Rev. A 100, 023810 (2019).

[33] B. Zhen, C. W. Hsu, L. Lu, A. D. Stone, and M. Soljačič, "Topological nature of optical bound states in the continuum," Phys. Rev. Lett. 113, 257401 (2014).

[34] E. N. Bulgakov and D. N. Maksimov, "Bound states in the continuum and polarization singularities in periodic arrays of dielectric rods," Phys. Rev. A 96, 063833 (2017).

[35] L. Yuan and Y. Y. Lu, "Bound states in the continuum on periodic structures: perturbation theory and robustness," Opt. Lett. 42(21) 4490-4493 (2017).

[36] A. Kodigala, T. Lepetit, Q. Gu, B. Bahari, Y. Fainman, and B. Kanté, "Lasing action from photonic bound states in continuum," Nature 541, 196-199 (2017).

[37] S. Romano, A. Lamberti, M. Masullo, E. Penzo, S. Cabrini, I. Rendina, and V. Mocella, "Optical biosensors based on photonic crystals supporting bound states in the continuum," Materials 11, 526 (2018).

[38] F. Yesilkoy, E. R. Arvelo, Y. Jahani, M. Liu, A. Tittl, V. Cevher, Y. Kivshar, and H. Altug, "Ultrasensitive hyperspectral imaging and biodetection enabled by dielectric metasurfaces," Nature Photonics 13, 390-396 (2019).

[39] J. M. Foley, S. M. Young, and J. D. Phillips, "Symmetryprotected mode coupling near normal incidence for narrow-band transmission filtering in a dielectric grating," Phys. Rev. B 89, 165111 (2014).

[40] X. Cui, H. Tian, Y. Du, G. Shi, and Z. Zhou, "Normal incidence filter using symmetry-protected modes in dielectric subwavelength gratings," Sci. Rep. 6, 36066 (2016)

[41] S. Han, L. Cong, Y. K. Srivastava, B. Qiang, M. V. Rybin, A. Kumar, R. Jain, W. X. Lim, V. G. Achanta, S. S. Prabhu, Q. J. Wang, Y. S. Kivshar, and R. 
Singh, "All-dielectric active terahertz photonics driven by bound states in the continuum," Advanced Materials, 31, 1901921 (2019).

[42] L. Yuan and Y. Y. Lu, "Diffraction of plane waves by a periodic array of nonlinear circular cylinders," Phys. Rev. A 94, 013852 (2016).

[43] L. Yuan and Y. Y. Lu, "Strong resonances on periodic arrays of cylinders and optical bistability with weak incident waves," Phys. Rev. A 95, 023834 (2017)

[44] E. N. Bulgakov and D. N. Maksimov, "Light enhancement by quasi-bound states in the continuum in dielectric arrays," Opt. Express 25(13), 14134-14147 (2017)

[45] L. Yuan and Y. Y. Lu, "Bound states in the continuum on periodic structures surrounded by strong resonances," Phys. Rev. A 97, 043828 (2018)

[46] K. Koshelev, S. Lepeshov, M. Liu, A. Bogdanov, and Y. Kibshar, "Asymmetric metasurfaces with high- $Q$ res- onances governed by bound states in the contonuum," Phys. Rev. Lett. 121, 193903 (2018)

[47] Z. Hu and Y. Y. Lu, "Resonances and bound states in the continuum on periodic arrays of slightly noncircular cylinders," J. Phys. B: At. Mol. Opt. Phys. 51, 035402 (2018).

[48] J. Jin, X. Yin, L. Ni, M. Soljacic, B. Zhen, and C. Peng, "Topologically enable unltra-high- $Q$ guided resonances robust to out-of-plane scattering," arXiv: 1812.00892v1

[49] L. Yuan and Y. Y. Lu, "Unidirectional reflectionless transmission for two-dimensional $\mathcal{P} \mathcal{T}$-symmetric periodic structures,", Phys. Rev. A

[50] L. N. Trefethen, "Spectral methods in MATLAB," Society for Industrial and Applied Mathematics (2000).

[51] Y. Huang and Y. Y. Lu, "Scattering from periodic arrays of cylinders by Dirichlet-to-Neumann maps," J. Lightwave Technol. 24, 3448-3453 (2006). 